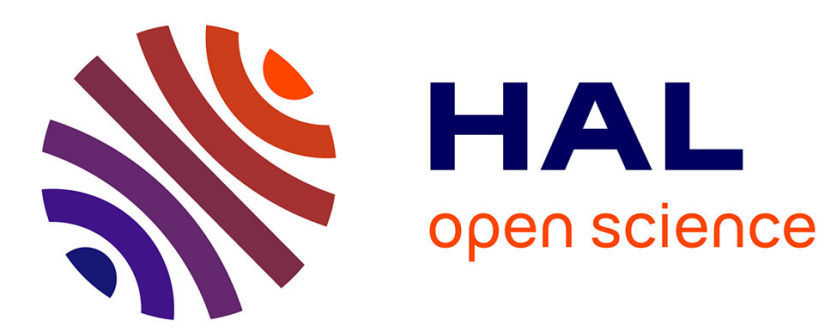

\title{
A mathematical framework for the control of piecewise-affine models of gene networks
}

\author{
Etienne Farcot, Jean-Luc Gouzé
}

\section{To cite this version:}

Etienne Farcot, Jean-Luc Gouzé. A mathematical framework for the control of piecewise-affine models of gene networks. Automatica, 2008, 44 (9), pp.2326-2332. 10.1016/j.automatica.2007.12.019 . hal00831803

\section{HAL Id: hal-00831803 \\ https://inria.hal.science/hal-00831803}

Submitted on 7 Jun 2013

HAL is a multi-disciplinary open access archive for the deposit and dissemination of scientific research documents, whether they are published or not. The documents may come from teaching and research institutions in France or abroad, or from public or private research centers.
L'archive ouverte pluridisciplinaire HAL, est destinée au dépôt et à la diffusion de documents scientifiques de niveau recherche, publiés ou non, émanant des établissements d'enseignement et de recherche français ou étrangers, des laboratoires publics ou privés. 


\title{
A mathematical framework for the control of piecewise affine models of gene networks *
}

\author{
Etienne Farcot ${ }^{\mathrm{a}}$, Jean-Luc Gouzé ${ }^{\mathrm{a}}$ \\ ${ }^{a}$ COMORE INRIA, Unité de recherche Sophia Antipolis, 2004 route des Lucioles, BP 93, 06902 Sophia Antipolis, France
}

\begin{abstract}
This article introduces results on the control of gene networks, in the context of piecewise-affine models. We propose an extension of this well-documented class of models, where some input variables can affect the main terms of the equations, with a special focus on the case of affine dependence on inputs. Some generic control problems are proposed, which are qualitative, respecting the coarse-grained nature of piecewise-affine models. Piecewise constant feedback laws that solve these control problems are characterized in terms of affine inequalities, and can even be computed explicitly for a subclass of inputs. The latter is characterized by the condition that each state variable of the system is affected by at most one input variable. These general feedback laws are then applied to a two dimensional example, consisting in two genes inhibiting each other. This example has been observed in real biological systems, and is known to present a bistable switch for some parameter values. Here, the parameters can be controlled, allowing to express feedback laws leading to various behaviours of this system, including bi-stability as well as situations involving a unique global equilibrium.
\end{abstract}

Key words: Gene network models ; Piecewise affine systems ; Qualitative control

\section{Introduction}

This work deals with control theoretic aspect of a class of piecewise-affine differential equations, which has been introduced in the 1970's by Leon Glass [17] to model genetic and biochemical interaction networks. Various aspects of the autonomous dynamics of these equations have been studied by different authors, e.g. $[10,14,17,18]$. Besides theoretical aspects, they have been used also as models of concrete biological systems [8,26], and efficient procedures have been proposed to identify their parameters $[9,25]$. This proves their possible use as models guiding experimental researches on gene regulatory networks. Such experiments have been carried out extensively during the recent years, often on large scale systems, thanks to the extraordinary developments of large throughput methods used in the investigation of biochemical systems.

Furthermore, recent advances have shown that such networks may not only be studied and analyzed on existing

\footnotetext{
* This paper was not presented at any IFAC meeting. Corresponding author E. Farcot. Tel. +33492387185. Fax +3392387858 .
}

Email addresses: etienne.farcot@sophia.inria.fr (Etienne Farcot), gouze@sophia.inria.fr (Jean-Luc Gouzé). biological species, but also synthesized in labs $[2,13,16]$. This latter aspect strongly motivates the elaboration of a control theory for these systems $[1,20,24]$. This work is an attempt in this direction: piecewise affine models are treated in the case where production and degradation terms can be modified during an experiment, a fact we model by introducing continuous input variables to control the system.

The biological interpretation of these inputs is that an additional biochemical compound is added to the system, or some physical parameter is changed. Such a modification may then activate, or inhibit the production or degradation rates of species involved in the system. Among concrete realizations, some specific inhibitors or activators could be introduced in the system, like is done in synthetic biology [24]. Other techniques, such as directed mutagenesis, the use of interfering RNA (siRNA and miRNA) [21,22], could also modify production or degradation rates. More radically, gene knock-in or knock-out techniques could be handled within this framework, their on/off nature being described by restricting the input values to a discrete set.

Being piecewise linear, the models that are studied here can be seen as particular switched or hybrid systems. As such, they might be handled using more general tools, tailored for these broad classes [12,23,27]. However, whereas a specialization of these techniques would 
concern steady states and their stability, we propose more specific feedback laws, notably to control trajectories across a prescribed sequence of boxes, which might include oscillatory behaviours. Moreover, we take advantage of the specific form of systems we consider.

Other comparable techniques are those developed in a series of papers treating multi-affine dynamical systems defined on rectangles $[3,4,19]$. The starting point of these different methods and algorithms is the control of all trajectories of a multi-affine dynamical system toward a specified facet of a full dimensional rectangle in state space. The input values have to satisfy a system of $2^{n-1}$ inequalities (one for each vertex of the exit facet). Being affine in rectangular regions of state space, the systems introduced here could be handled with these techniques. However, our method requires to check a number of inequalities that is proportional to $n$, and which can even be solved explicitely in some cases, improving drastically the complexity of a blind application of more general techniques.

The paper is organized in three main sections. In section 2, the investigated model is introduced. Then in section 3, we formulate generic control problems, whose solutions are characterized in section 3.3. In section 4 we illustrate the method on a classical two-dimensional example: the toggle switch, showing notably how to induce bi-stability in this system. A final section discusses the results, and the possible outcomes of this work.

\section{Piecewise Affine Models}

\subsection{The Autonomous Case}

The usual form of piecewise affine gene network models may be:

$$
\frac{d x}{d t}=\kappa(x)-\Gamma(x) x .
$$

$\kappa(x) \in \mathbb{R}_{+}^{n}$ is a production term, and $\Gamma(x)$ a diagonal matrix with positive diagonal entries, representing degradation rates of system. Both are piecewise constant with a rectangular underlying partition, see below.

The fact that $\kappa$ and the $\gamma_{i}$ 's are piecewise constant of $x$ is due to the switch-like nature of the feedback regulation in gene networks. The variable $x_{i}$ is a concentration (of mRNA or of protein), representing the expression level of the $i$ th gene among $n$, and ranges in some interval of the form $\left[0, \max _{i}\right]$. When $x_{i}$ reaches a threshold value, some other gene in the network, say gene number $j$, is suddenly activated (resp. inhibited), and thus expressed with a different production rate : the value of $\kappa_{j}$ (resp. $\gamma_{j}$ ) changes. For each $i \in\{1 \cdots n\}$ there is thus a finite set of threshold values :

$$
\Theta_{i}=\left\{\theta_{i}^{1} \ldots \theta_{i}^{q_{i}-1}\right\}
$$

supposed ordered: $0<\theta_{i}^{1}<\cdots<\theta_{i}^{q_{i}-1}<\max _{i}$. Although the extreme values may not be crossed, we denote $\theta_{i}^{0}=0$, and $\theta_{i}^{q_{i}}=\max _{i}$ by convention.
By definition of $\kappa$ and $\Gamma$, the axes of the state space will be partitioned into open segments between thresholds. Since the extreme values will not be crossed by the flow, the first and last segments include one of their endpoints:

$$
\begin{array}{r}
\mathcal{D}_{i} \in \Theta_{i} \cup\left\{\left[\theta_{i}^{0}, \theta_{i}^{1}\right),\left(\theta_{i}^{q_{i}-1}, \theta_{i}^{q_{i}}\right]\right\} \cup \\
\left\{\left(\theta_{i}^{j}, \theta_{i}^{j+1}\right) \mid j \in\left\{1 \cdots q_{i}-2\right\}\right\}
\end{array}
$$

Each product $\mathcal{D}=\prod_{i=1}^{n} \mathcal{D}_{i}$ defines a rectangular domain, whose dimension is the number of non-singleton $\mathcal{D}_{i}$. When $\operatorname{dim} \mathcal{D}=n$, one usually says that it is a regulatory domain, or regular domain, and those domains with lower dimension are called switching domains, or singular domains, see [8]. In particular, singular domains of dimension $n-1$ are often called walls.

Let $\mathscr{D}_{r}$ and $\mathscr{D}_{s}$ denote the collections of regulatory and switching domains, respectively. The underlying regions in state space are denoted by $|\cdot|$, i.e. for example $\left|\mathscr{D}_{r}\right|=\bigcup_{\mathcal{D} \in \mathscr{D}_{r}} \mathcal{D}$ is the whole state space with all threshold hyperplanes removed. On this set, the dynamics will be called hereafter regular dynamics.

On $\left|\mathscr{D}_{s}\right|$ on the other hand, the flow is in general only defined in a weak sense, yielding what will be mentioned as the singular dynamics. In short, the latter is usually $[8,26]$ presented as a set-valued version of the regular dynamics, applying the technique developed by Filippov, and first applied to systems of the form (1) in [18]. We refer the interested reader to the mentioned literature for more thorough treatments of singular solutions. We just recall that walls such that flow lines are directed in opposite directions on their two sides are usually called white (resp. black) walls if repelling (resp. attracting). Moreover, it can be shown that the regular dynamics can be extended properly on any other wall then called transparent-, and thus on a dense subset of $\left|\mathscr{D}_{s}\right|$ in tame situations.

\subsubsection{Regular Dynamics.}

Regulatory domains form the main part of state space. Moreover, on any $\mathcal{D} \in \mathscr{D}_{r}, \kappa$ and $\Gamma$ are constant, and thus equation (1) is affine. Its solution is explicitly known:

$$
\forall i \quad \varphi_{i}(x, t)=x_{i}(t)=\frac{\kappa_{i}}{\gamma_{i}}+e^{-\gamma_{i} t}\left(x_{i}(0)-\frac{\kappa_{i}}{\gamma_{i}}\right),
$$

and is valid for all $t \in \mathbb{R}_{+}$such that $x(t) \in \mathcal{D}$. It follows immediately that

$$
\phi(\mathcal{D})=\left(\phi_{1}, \cdots, \phi_{n}\right)=\left(\frac{\kappa_{1}}{\gamma_{1}}, \cdots, \frac{\kappa_{n}}{\gamma_{n}}\right)
$$

is an attractive equilibrium point for the flow (3). If it does not belong to $\mathcal{D}$, it is not a real equilibrium for the system (1), since the flow will reach the boundary 
$\partial \mathcal{D}$ in finite time. At that time, the value of $\kappa$ or $\Gamma$ will change, and that of $\phi$ accordingly. The point $\phi(\mathcal{D})$ is often called focal point of the domain $\mathcal{D}$.

A convenient notation will be the following: each domain $\mathcal{D} \in \mathscr{D}_{r}$, with closure of the form $c \ell(\mathcal{D})=$ $\prod_{i=1}^{n}\left[\theta_{i}^{a_{i}-1}, \theta_{i}^{a_{i}}\right]$, can be represented by the integer vector $a=\left(a_{1}, \ldots, a_{n}\right)$. Then one defines $\mathcal{V}=$ $\prod_{i=1}^{n}\left\{1 \cdots q_{i}\right\} \simeq \mathscr{D}_{r}$. Regular domains and their representatives in $\mathcal{V}$ will be constantly identified, leading to talk about some "domain $a$ ", or noting focal points $\phi(a)$. Then, a discretizing mapping $\mathrm{d}=\left(\mathrm{d}_{1} \ldots \mathrm{d}_{n}\right):\left|\mathscr{D}_{r}\right| \rightarrow \mathcal{V}$ associates to a point lying inside a regular domain the discrete representative of this domain. One can also naturally define a set of transitions $\mathcal{E} \subset \mathcal{V} \times \mathcal{V}$, where $(a, b) \in \mathcal{E}$ iff some continuous trajectory successively crosses domains $a$ and $b$. Hence one gets a transition graph, defined as the pair $\mathrm{TG}=(\mathcal{V}, \mathcal{E})$. It can be shown that the following is appropriate, see e.g. [15].

\section{Definition 1 (transition graph)}

$\mathrm{TG}=(\mathcal{V}, \mathcal{E})$, where $\mathcal{V}=\prod_{i=1}^{n}\left\{1 \cdots q_{i}\right\}$, and $(a, b) \in \mathcal{E}$ if and only if $b=a$ and $\mathrm{d}(\phi(a))=a$, or $b \in\left\{a+\varepsilon_{i} \mathbf{e}_{i} \mid \varepsilon_{i}=\right.$ $\left.\operatorname{sign}\left(\mathrm{d}_{i}(\phi(a))-a_{i}\right) \neq 0\right\}$, where $\mathbf{e}_{i}$ is the ith vector of the canonical basis of $\mathbb{R}^{n}$.

Observe that each vertex in TG may usually have several outgoing edges, i.e. this is a non deterministic graph. Although in general many paths in TG do not represent any continuous trajectory, it can be shown that every regular solution of (1) admits a well-defined representative as an infinite path in TG, see [14]. An important point is that the transitions between adjacent regular domains are determined by the position of focal points.

The following hypothesis will be useful:

H1 $\forall \mathcal{D} \in \mathscr{D}_{r}, \quad \phi(\mathcal{D}) \in\left|\mathscr{D}_{r}\right|$.

H1 implies that $\prod_{i}\left[0, \max _{i}\right]$ is positively invariant, and thus can be considered as the only region where relevant dynamics take place. Moreover, focal points on threshold hyperplanes represent a technical complication, and do not improve the model.

\subsection{Piecewise Constant Feedback Control}

Using inputs, the following form is the most obvious:

$$
\frac{d x}{d t}=\kappa(x, u)-\Gamma(x, u) x
$$

where $u \in \mathcal{U}=\prod_{j=1}^{p}\left[0, U_{j}\right]$, since inputs are concentrations or physical parameters: one will deal with bounded variables only. That is, $U_{j}$ is the upper bound of input coordinate $u_{j}$. If some input quantities are essentially discrete (e.g. a gene is turned on, or off), one may consider finite subsets of the intervals $\left[0, U_{j}\right]$, apply the treatments that we propose, and check afterwards whether at least one of the discrete values is also a solution.
In the present work, we focus on piecewise constant feedback control laws: $u=u(x)$, and the restriction $\left.u\right|_{\mathcal{D}}$ is constant for each $\mathcal{D} \in \mathscr{D}_{r}$. This relies on the assumption that threshold crossings, i.e. switchings, can be detected accurately [9]. A consequence of this choice is that the input may be unambiguously defined as the composition of $d$ and a map $\mathcal{V} \rightarrow \mathcal{U}$, see section 2.1 for the notations. We shall often identify $u$ and this latter map.

Whatever the precise form of $\kappa(x, \cdot)$ and $\Gamma(x, \cdot)$, seen as functions of the input $u$, the choice of a feedback loop depending on the regulatory domain of the current state, rather than on its precise quantitative value, has nice consequences on the dynamics. Actually, for any fixed feedback law $u$, the behaviour of the system is exactly similar to the autonomous case, since $u$ is constant in each regular domain. The focal point of such a domain is now of the form :

$$
\phi(a, u(a))=\left(\frac{\kappa_{1}(a, u(a))}{\gamma_{1}(a, u(a))} \cdots \frac{\kappa_{n}(a, u(a))}{\gamma_{n}(a, u(a))}\right),
$$

where the vector $u(a)$ has a fixed value, that can be chosen according to some specified purpose.

The controllable focal set is the whole set $\phi(a, \mathcal{U})$ in which focal points can be chosen, when $u$ is varied. Although the shorter term focal set is often employed when using Filippov solutions [18], with a quite different meaning, we shall sometimes use it as an abbreviation, since it is not ambiguous in the present study.

$\mathcal{U}$ being compact and $\phi(a, \cdot)$ continuous, the focal set will generally be a $p$-dimensional compact subspace of $\mathbb{R}_{+}^{n}$. The possible successors of the domain $\mathcal{D}_{a}$ are then the regular domains indexed by:

$$
\mathcal{S}(a)=\left\{a+\operatorname{sign}(b-a) \mid \mathcal{D}_{b} \cap \phi(a, \mathcal{U}) \neq \varnothing\right\},
$$

where sign $: \mathbb{R}^{\mathrm{n}} \rightarrow\{-1,0,1\}^{\mathrm{n}}$ is the coordinate-wise signum function, used here to consider successors only among domains adjacent to $\mathcal{D}_{a}$.

To be more explicit, one will assume now that both $\kappa$ and $\Gamma$ are affine functions of $u$, in each regular domain. In this case we use the following notation:

$$
\begin{cases}\kappa_{i}(x, u)=\sum_{j=1}^{p} \kappa_{i}^{j}(x) u_{j}+\kappa_{i}^{0}(x) & i \in\{1 \cdots n\} \\ \gamma_{i}(x, u)=\sum_{j=1}^{p} \gamma_{i}^{j}(x) u_{j}+\gamma_{i}^{0}(x) & i \in\{1 \cdots n\}\end{cases}
$$

with piecewise constant functions $\kappa_{i}^{j}$ and $\gamma_{i}^{j}$. We use $u$ instead $u(x)$ to lighten notations, since the feedback is not our main interest presently. Eq. (7) can be interpreted as follows, considering production rates, the case of degradation rates being identical. For each $i \in\{1 \cdots n\}$, and for a fixed $x, \kappa_{i}(x, \cdot)$ is a function of $u$, and has the biological meaning of a production rate. Then, the $\kappa_{i}^{j}$ functions can be understood as the coefficients of $\kappa_{i}$, 
once it is assumed that the latter is affine in $u$. Thus, these $\kappa_{i}^{j}$ functions may be interpreted as the relative strengths of the different inputs, in their influence on $\kappa_{i}$. In some sense, this choice is mostly relevant in the case when there is no interaction between the inputs.

Let us denote $u^{0}$ the input value in the autonomous case. Its coordinates may be nonzero, especially when they represent some physical parameter, which should be possibly decreased by the user. Also, production or degradation rates should not always be increasing functions of the input, i.e. one must allow negative coefficients in (7). However, one must also satisfy $\gamma_{i}(x, u)>0$ for all $i$, because biochemical compounds always degrade, and $\kappa_{i}(x, u) \geqslant 0$, since otherwise the concentration $x_{i}$ could reach negative values. All these conditions are satisfied under simple restrictions on the parameters in (7).

H2 For all $i \in\{1 \cdots n\}$, and all $a \in \mathcal{V}, \gamma_{i}^{0}(a)>0$, $\kappa_{i}^{0}(a) \geqslant 0$. Moreover

$$
\left\{\begin{array}{l}
\sum_{\substack{j \in\{1 \cdots p\} \\
\gamma_{i}^{j}(a)<0}} \gamma_{i}^{j}(a) U_{j}>-\gamma_{i}^{0}(a) \\
\sum_{\substack{j \in\{1 \cdots p\} \\
\kappa_{i}^{j}(a)<0}} \kappa_{i}^{j}(a) U_{j} \geqslant-\kappa_{i}^{0}(a)
\end{array}\right.
$$

Actually, the left-hand sides above are easily seen to be the infimum, among all inputs, of the linear parts of affine functions $\kappa_{i}$ and $\gamma_{i}$.

Equation (7) may be put in matrix form:

$$
\left\{\begin{array}{l}
\Gamma(x, u)=\operatorname{diag}\left(\Gamma(x) u+\gamma^{0}(x)\right) \\
\kappa(x, u)=\kappa(x) u+\kappa^{0}(x)
\end{array}\right.
$$

where $\kappa \in \mathbb{R}^{n \times p}, \kappa^{0} \in \mathbb{R}_{+}^{n \times 1}, \Gamma \in \mathbb{R}^{n \times p}$ and $\gamma^{0} \in \mathbb{R}_{+}^{n \times 1}$, omitting $x$.

Being still more specific, we may further assume that at most one input variable is acting on each state variable. This could correspond to the use of biochemical inputs interacting selectively, with state variables affected only by some specific input. Then, either the influence of $u$ appears on the production term, or on the decay rate of each $x_{i}$. This can be formulated using two functions $\sigma, \varsigma$ : $\{1 \cdots n\} \rightarrow\{1 \cdots p\}$, such that for each $i$ in $\{1 \cdots n\}$, all coefficients in the matrices $\kappa(x)$ and $\Gamma(x)$ are zero, except maybe $\kappa_{i}^{\sigma(i)}$ and $\gamma_{i}^{\varsigma(i)}$.

$$
\left\{\begin{array}{l}
\kappa_{i}(x, u)=\kappa_{i}^{\sigma(i)}(x) u_{\sigma(i)}+\kappa_{i}^{0}(x) \\
\gamma_{i}(x, u)=\gamma_{i}^{\varsigma(i)}(x) u_{\varsigma(i)}+\gamma_{i}^{0}(x) .
\end{array}\right.
$$

and moreover, $\forall i \in\{1 \cdots n\}, \forall a \in \mathcal{V}$

$$
\kappa_{i}^{\sigma(i)}(a)=0 \text { or } \gamma_{i}^{\varsigma(i)}(a)=0
$$

Note that both may still be zero. In order to clarify further discussions, we now give a name to the two classes of inputs we have presented. These are the only classes of systems that we shall consider in the following.

\section{C1 (affine dependance on inputs)}

Refers to systems of the form (4), where production and degradation terms are all affine functions of $u$, of the form (7).

\section{C2 (single input per variable)}

Refers to systems of the class $\mathbf{C 1}$, where at most one input affects each variable $x_{i}$, i.e. (9) and (10) are satisfied.

\section{Specific Control Problems}

\subsection{Discrete Mapping of Systems with Input}

Once a feedback law is chosen, one has seen that a system of the form (4) is equivalent to an autonomous system of the form (1). Then, a discrete system can be constructed, as described in the autonomous case. Refining the definition of discrete successors provided in (6) let us define $\mathcal{S}(a, u)=a+\operatorname{sign}(\mathrm{d}(\phi(a, u))-a)$, and denote $\mathcal{S}_{i}(a, u)$ its coordinates. Then, varying a feedback law amounts to varying $u(a)$ in the whole set $\mathcal{U}$, for all $a$. Now, if $u$ is such a feedback law, let us define $\mathrm{TG}(u)=(\mathcal{V}, \mathcal{E}(u))$, where $\mathcal{V}$ is the same as in the autonomous case and, following definition 1 :

$$
\begin{array}{r}
\mathcal{E}(u)=\bigcup_{a \in \mathcal{V}}\{a\} \times\left\{a+\varepsilon_{i} \mathbf{e}_{i} \mid i=1 \ldots n,\right. \\
\left.\varepsilon_{i}=\operatorname{sign}\left(\mathcal{S}_{i}(a, u)-a_{i}\right)\right\},
\end{array}
$$

Then, a generic control problem can be formulated in global terms, involving a desirable transition graph.

Problem 1 (global control problem) Let $\mathrm{TG}^{\star}$ be a transition graph. Find a feedback law $u: \mathcal{V} \rightarrow \mathcal{U}$ such that $\mathrm{TG}(u)=\mathrm{TG}^{\star}$.

There are many ways to choose a target transition graph $\mathrm{TG}^{\star}$. For instance, it can be defined to satisfy certain properties, notably expressed in some temporal logic [5]. Note that transition graphs are huge in general, $\mathcal{V}$ being of exponential cardinality. Anyway, the target transition graph $\mathrm{TG}^{\star}$ may differ from the autonomous graph $\mathrm{TG}\left(u^{0}\right)$ only on a subset of edges. Hence, problem 1 includes local versions, where the feedback law is only sought on a subset $\mathcal{V}^{\star}$ of the vertices $\mathcal{V}$. In this case, the edges of $\mathrm{TG}^{\star}$ differ from that of $\mathrm{TG}\left(u^{0}\right)$ only when their source is in $\mathcal{V}^{\star}$. Now, we elucidate the most elementary local problem, where $\mathcal{V}^{\star}$ is a single vertex. 


\subsection{Control of a Single Box}

The control of a one element vertex set $\mathcal{V}^{\star}=\{a\}$ corresponds, in a continuous state space, to the control of a single regular domain $\mathcal{D}_{a}$. We have seen in sections 2 and 2.2 that the dynamics in such domains is essentially determined by a focal point $\phi(a, u(a))$. If it belongs to $\mathcal{D}_{a}$, it is an attracting equilibrium, whereas is $\phi(a, u(a)) \notin \mathcal{D}_{a}$, all trajectories escape from $\mathcal{D}_{a}$ in finite time. More precisely, they can escape via a given wall $W_{i}(a) \subset c \ell\left(\mathcal{D}_{a}\right) \cap\left\{x \mid x_{i}=\theta_{i}\right\}$, where $\theta_{i}=\theta_{i}^{a_{i}-1}$ (resp. $\left.\theta_{i}^{a_{i}}\right)$, if and only if $\phi(a)<\theta_{i}^{a_{i}-1}$ (resp. $\phi(a)>\theta_{i}^{a_{i}}$ ). This is exactly the property used to set up definition 1 , and comes from (3). Observe moreover that either all trajectories that reach a given wall from $\mathcal{D}_{a}$ escape this domain, or none of them do.

Hence, the control of a single box can be written as

$$
\exists u \in \mathcal{U}, \forall i \in\{1 \cdots n\}, \quad \theta_{i}^{-}<\phi_{i}(a, u)<\theta_{i}^{+},
$$

where the thresholds $\theta_{i}^{ \pm} \in \Theta_{i}$ are generic notations, and inequalities shall be weakened when concerning the boundaries of the whole domain. The remaining work is thus to define an input $u$ such that the above system of inequalities is satisfied. In words, (12) states the existence of an input $u$ such that $\phi_{i}(a, u)$ belongs to rectangular union of regular domains. Two special cases of (12) are particularly interesting, and shown on figure 1 .

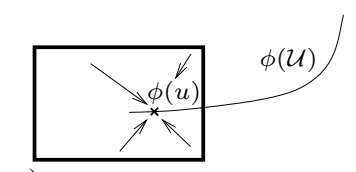

a)

b)

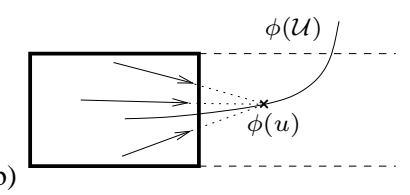

Fig. 1. Among all points in the focal set $\phi(\mathcal{U})$, one has to chose a particular $u$. Case a) is to make a region invariant, while in b), one has to find an input $u$ such that $\phi(u)$ is situated 'behind' a single facet of the box under consideration.

\subsubsection{Control Making a Regular Region Invariant.}

The first problem corresponds to a situation where $\mathcal{D}_{a}$ represents a beneficial situation for the system. Then, the only possibility to preclude a qualitative change in behaviour at a state $a$, with a constant input $u(a)$ on $\mathcal{D}_{a}$, is to force $\phi(a, u(a))$ to be a stable equilibrium. In other words one has to find a constant $u=u(a)$ such that $\phi(a, u) \in \mathcal{D}_{a}$. This is equivalent to (12), with $\theta_{i}^{-}=\theta_{i}^{a_{i}-1}$ and $\theta_{i}^{+}=\theta_{i}^{a_{i}}$. See figure $\left.1, \mathrm{a}\right)$.

\subsubsection{Escaping from a Region through a Single Facet.}

Another control problem is to escape from $\mathcal{D}_{a}$, with the additional condition that this happens through a single prescribed wall. The main argument in favor of this more particular control problem is the fact that it leads to a transition graph $\mathrm{TG}(u)$ where $a$ admits a unique successor. We have seen in section 2.1 that some paths in TG do not represent a trajectory of the initial system. Said in terms of simulation relation [6,3], TG simulates the regular dynamics, while the converse does not hold in general. If all vertices in TG are the source of at most one arrow, then the converse holds. This fact is quite intuitive since, given an initial domain, all trajectories will follow a uniquely defined sequence of domains. One shall say in this case that the continuous system and its discrete quotient are bisimilar. For a general definition of this notion, see also $[6,3]$. This property of TG is pictured for a single box in figure $1, b)$. Given an escaping direction $i$ this fits (12), with $\theta_{j}^{-}=\theta_{j}^{a_{j}-1}$ and $\theta_{j}^{+}=\theta_{j}^{a_{j}}$ for $j \neq i$, and $\theta_{i}^{-}=\theta_{i}^{a_{i}}, \theta_{i}^{+}=\theta_{i}^{q_{i}}$, or $\theta_{i}^{-}=\theta_{i}^{0}, \theta_{i}^{+}=\theta_{i}^{a_{i}-1}$ depending on the escaping wall.

\subsection{Generic Control Law for a Single Box}

We now characterize the inputs $u$ solving (12). Since the domain $a$ will be clear, we omit in functions $\kappa_{i}^{j}$ and $\gamma_{i}^{j}$ hereafter. We state results without proofs. The latter are not difficult, and can be found in [15].

Before all, remark that if $\phi_{i}=0$, i.e. $\kappa_{i}=0$, for some $i \in\{1 \cdots n\}$, then either $\theta_{i}^{-}>0$, and the problem (12) admits no solution, or $\theta_{i}^{-}=0$, and any $u \in \mathcal{U}$ solves this problem for the coordinate $i$. In the following we ignore this special case.

Proposition 1 Consider a system of the class $\mathbf{C 1}$. Define $T^{ \pm}=\operatorname{diag}\left(\theta_{1}^{ \pm} \cdots \theta_{n}^{ \pm}\right) \in \mathbb{R}^{n \times n}$.

Then, any $u \in \mathcal{U}$ satisfying the system below is a solution of problem (12).

$$
\left\{\begin{array}{l}
\left(\kappa-T^{-} \Gamma\right) u>T^{-} \gamma^{0}-\kappa^{0} \\
\left(\kappa-T^{+} \Gamma\right) u<T^{+} \gamma^{0}-\kappa^{0}
\end{array}\right.
$$

Note that the condition $u \in \mathcal{U}$ can also be written as pairs of inequalities of the form $0 \leqslant u_{j} \leqslant U_{j}$.

For systems in the class $\mathbf{C 2}$, the inequalities can be solved by hand, and thus an explicit input may be expressed. Remind that, for these systems, there are two functions $\sigma, \varsigma:\{1 \cdots n\} \rightarrow\{1 \cdots p\}$, defining the input acting respectively on the production and degradation rates of a given variable, see (9) and (10). The last of these two equations imposes that for each $i$, at least one of the two coefficients $\kappa_{i}^{\sigma(i)}$ and $\gamma_{i}^{\varsigma(i)}$ is zero. Accordingly, we introduce $S:\{1 \cdots n\} \rightarrow\{0 \cdots p\}$, which is defined by $S(i)=\sigma(i)$ (resp. $\varsigma(i))$ if $\kappa_{i}^{\sigma(i)} \neq 0$ (resp. $\gamma_{i}^{\varsigma(i)} \neq 0$ ), and $S(i)=0$ if $\kappa_{i}^{\sigma(i)}=\gamma_{i}^{\varsigma(i)}=0$. Let us also define, for $i \in\{1 \cdots n\}$, the bounds of $\phi_{i}(\mathcal{U}): \phi_{i}^{-}=\min \left\{\phi_{i}(0), \phi_{i}\left(U_{S(i)}\right)\right\}$, and $\phi_{i}^{+}=$ $\max \left\{\phi_{i}(0), \phi_{i}\left(U_{S(i)}\right)\right\}$. Actually, for the class C2, $\phi_{i}$ is of one of these two forms: $\frac{\kappa_{i}^{\sigma(i)} u_{\sigma(i)}+\kappa_{i}^{0}}{\gamma_{i}^{0}}$ or $\frac{\kappa_{i}^{0}}{\gamma_{i}^{\varsigma(i)} u_{\varsigma(i)}+\gamma_{i}^{0}}$, 
which are always monotonic. Thus the bounds of their image are the images of their domain's bounds. Clearly, the bounds of the interval $\phi_{i}(\mathcal{U}) \cap\left(\theta_{i}^{-}, \theta_{i}^{+}\right)$are $\max \left\{\phi_{i}^{-}, \theta_{i}^{-}\right\}$and $\min \left\{\phi_{i}^{+}, \theta_{i}^{+}\right\}$. Now in direction $i$, let $m_{i}^{-}$and $m_{i}^{+}$be respectively the min and max of the pair $\phi_{i}^{-1}\left(\max \left\{\phi_{i}^{-}, \theta_{i}^{-}\right\}\right)$and $\phi_{i}^{-1}\left(\min \left\{\phi_{i}^{+}, \theta_{i}^{+}\right\}\right)$. It follows that $\left(m_{i}^{-}, m_{i}^{+}\right) \subset\left[0, U_{S(i)}\right]$ is exactly the possibly empty interval of input coordinates that solve the control problem in direction $i$. In practice, these numbers may be easily expressed since $\phi_{i}$ is known explicitly. Then, for $q \in\{1 \cdots p\}$, define $\mu_{q}^{-}=\max _{i \in S^{-1}(q)} m_{i}^{-}$, and $\mu_{q}^{+}=\min _{i \in S^{-1}(q)} m_{i}^{+}$. By construction, for any $u_{q} \in\left(\mu_{q}^{-}, \mu_{q}^{+}\right)$and $i$ such that $S(i)=q$, one has $\phi_{i}\left(u_{q}\right) \in\left(\theta_{i}^{-}, \theta_{i}^{+}\right)$as expected. Hence,

Proposition 2 Consider a system belonging to the class C2. Let $\mu_{q}^{-}$and $\mu_{q}^{+}$be the quantities defined in the previous discussion. Then, the set of $u$ that solve inequalities (12) is exactly $\prod_{q=1}^{p}\left(\mu_{q}^{-}, \mu_{q}^{+}\right)$. Hence (12) admit a solution if, and only if $\mu_{q}^{-}<\mu_{q}^{+}$for all $q \in\{1 \cdots p\}$.

\section{The Toggle Switch Example}

Let us illustrate the methods of this paper on a system of two genes inhibiting each other. Its biological function is that of a switch between two steady states, each being a long-term, permanent response to some transient induction. This is the extensively studied toggle switch, which notably serves as a building block for larger biological circuits $[16,20,24]$. Here we suppose that a scalar input can affect the decay rates:

$$
\left\{\begin{array}{l}
\frac{d x_{1}}{d t}=\lambda_{1}^{1} \mathrm{~s}^{-}\left(x_{2}, \theta_{2}^{1}\right)+\lambda_{1}^{0}-\left(\gamma_{1}^{1} u+\gamma_{1}^{0}\right) x_{1} \\
\frac{d x_{2}}{d t}=\lambda_{2}^{1} \mathrm{~s}^{-}\left(x_{1}, \theta_{1}^{1}\right)+\lambda_{2}^{0}-\left(\gamma_{2}^{1} u+\gamma_{2}^{0}\right) x_{2}
\end{array},\right.
$$

where $\mathrm{s}^{-}(x, \theta)$ is the decreasing Heaviside (or step) function, which is 0 if $x>\theta$ and 1 if $x \leqslant \theta$.

We suppose that the following holds: $\frac{\lambda_{i}^{0}}{\gamma_{i}^{0}+\gamma_{i}^{1} u_{i}^{0}}<\theta_{i}^{1}$ for $i \in\{1,2\}$, and $\frac{\lambda_{1}^{0}+\lambda_{1}^{1}}{\gamma_{1}^{0}+\gamma_{1}^{1} u_{1}^{0}}<\theta_{1}^{1}, \frac{\lambda_{2}^{0}+\lambda_{2}^{1}}{\gamma_{2}^{0}+\gamma_{2}^{1} u_{2}^{0}}>\theta_{2}^{1}$. This is tantamount to say that $\mathrm{TG}\left(u^{0}\right)$ presents a single equilibrium, at state 12 : it is the graph a), below.

Bi-stability is useful to many biological systems, like in lysis vs. lysogeny in the $\lambda$-phage [28], or the life vs. apoptosis network [11]. Although oversimplified compared to these examples, model (13) is typically the simplest one exhibiting bi-stability [7], serving our illustrative purpose. Hence, we want to solve the specific problem

Problem 2 (bi-stability) Consider (13) with parameters as in fig. 2, i.e. a single globally stable equilibrium in region 12 in the autonomous situation. Find some

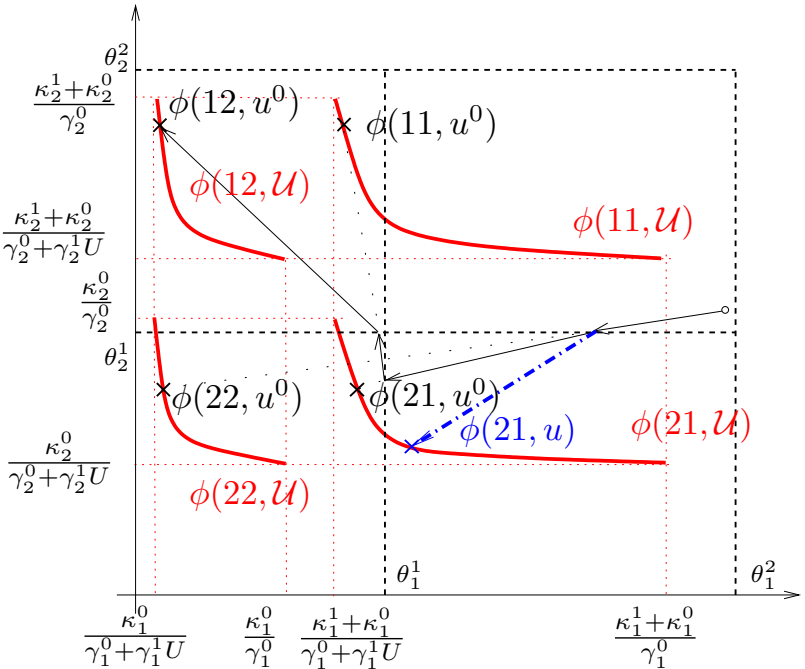

Fig. 2. There are 4 regular domains denoted $\frac{\mathcal{D}_{12} \mid \mathcal{D}_{22}}{\mathcal{D}_{11} \mid \mathcal{D}_{21}}$, bounded by dashed threshold lines, each having a piece of hyperbola as controllable focal set. The autonomous situation $u=u^{0}$ is such that a schematic trajectory starting in $\mathcal{D}_{22}$ (plain line) goes to $\mathcal{D}_{21}$, from which it escapes to $\mathcal{D}_{11}$, and finally to $\mathcal{D}_{12}$ which contains to the unique equilibrium of the system, $\phi\left(12, u^{0}\right)$. Changing the input value in $\mathcal{D}_{21}$ deviates this orbit to a newly created equilibrium, $\phi(21, u) \in \mathcal{D}_{21}$ (dashed-dotted line), hence inducing bi-stability.

$u: \mathcal{V} \rightarrow \mathcal{U}$ such that $\mathrm{TG}(u)$ is bi-stable, i.e. both regions 12 and 21 contain a stable equilibrium.

Bi-stability can be obtained among other ways by solving a local problem at state 21 , so that it becomes a fixed state, i.e. $\phi(21) \in \mathcal{D}_{21}$. Now, the state space of system (13) can be seen on figure 2. Since this system clearly belongs to class $\mathbf{C 2}$, it is possible to set up the inequalities of proposition 1, and then solve them by hand like in proposition 2. This is done in [15]. In this example however, the existence of solutions can also be seen on figure 2: the regular domains that are intersected by focal sets provide the controllable transitions. Hence it is possible to list all the controllable graphs. Then, according to a specific purpose those graphs that are satisfying may be chosen, and problem 1 be solved with such an objective graph. Let us just list all the possible transition graphs. To obtain a concise view of this graph, we represent self loops by filled squares, and omit the labels of vertices. Their disposition, yet, is in accordance with the geometry of state space: $\frac{12 \mid 22}{11 \mid 21}$. The autonomous case appears first, at $a$ ) a)<smiles>[AlH]1[AlH][SiH][AlH]1</smiles>

e)

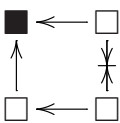

b)

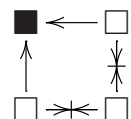

f)

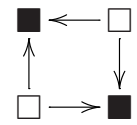

c)

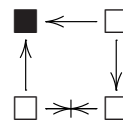

g)

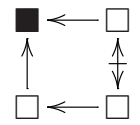

d)<smiles>[CH]1[Al][AlH][AlH]1</smiles>

h)

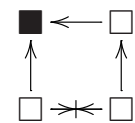


j)

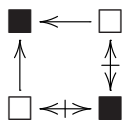

k)

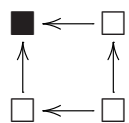

l)

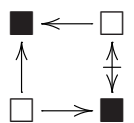

The doubly oriented arrows correspond to black or white walls in phase-space (cf. the end of section 2.1): black walls appear as $¥$ and white walls as $\langle 1>$. One can see that vertex 12 is always fixed, whatever the input value. Vertex 21, on the other hand, may be fixed or not, as we also have seen earlier.

Solution to problem 2: bi-stability may be ensured by applying one of the feedback laws leading to the transition graphs: $d), f), j$ ) and $l$ ). Among those, $d$ ) is the one illustrated in figure 2 , and $f$ ) provides the only bistable configuration without white wall.

Here are other examples of control problems and their solution

Problem 3 (bisimilarity) Consider (13) with a single autonomous equilibrium as in Prob 2. Find some $u: \mathcal{V} \rightarrow$ $\mathcal{U}$ such that TG $(u)$ is bisimilar to system (13).

Solution to problem 3: Recall that in our case, bisimilarity means that each node in TG admits at most one successor. This may be achieved by inputs associated to the graph $g$ ) and $j$ ). The first presents a single global equilibrium, while $j$ ) is bistable. Both present white walls.

Problem 4 (temporary switch) Consider (13) with parameters such that the autonomous situation is bistable, as in $f)$, and with an initial condition in state 21. Find some input law leading the system to state 12, but leaving the graph TG unaltered eventually.

Solution to problem 4: The state 12 is unreachable from 21 in $f$ ), but may be reached by applying a temporary input leading for example to the graph $a$ ): in this graph all trajectories from 21 enter 12 in finite time. When this state 12 is reached then, one may release $u$ to its autonomous value, leading back to the graph $f$ ) where 12 stays fixed for subsequent times. This type of temporary control aim at solving local problems, leaving the global graph TG unchanged eventually.

One may be surprised by the obvious asymmetry of the controllable graphs above: although both state variables are equivalent in (13), vertex 12 is always fixed, while 21 admits controllable successors. This is in fact a consequence of the special set of parameter values we have considered, chosen to match with figure 2 .

\section{Conclusion}

The goal of this paper is to provide a mathematical formulation for the control of gene networks. We focus on a class of piecewise affine models that have proved their efficiency in the last few years, formulated in (1), and we allow some parameters to be functions of an input $u$. Namely, both production and degradation terms are supposed to depend linearly on $u$, leading to equation (4). Given this class of systems, it appears relevant to look for piecewise constant feedback control laws. Notably, such input laws could be concretely implemented, provided threshold crossings can be detected. This latter fact is not out of reach today [9], and seems a reasonable ground for a methodological work like the present one. We then study the most natural control problems arising within this framework. They are expressed in terms of a discrete quotient of the system, presented as an oriented graph. Special attention is given to local control problems involving a single vertex in this graph, since they are the necessary first steps towards solving more global problems. This results in propositions 1 and 2 , which reduce any local problem to a system affine inequalities on the input. An important follow-up of this work would be the analysis of more global control problems. In particular, instead of targeting an explicit transition graph in problem 1, one may aim at satisfying a formal property, like bi-stability or bisimilarity as examplified in section 4. Systematizing this with the aid of tools from model checking might lead to efficient algorithms, able to deal with high-dimensional systems so that they satisfy a property expressed in some adapted temporal logic [6].

Acknowledgments. This work was partially supported by the European Commission, project Hygeia Nest-004995. The authors would like to thank D. Ropers and H. de Jong, from INRIA, for their helpful advice, and the anonymous referees for their judicious remarks.

\section{References}

[1] E. Andrianantoandro, S. Basu, D.K. Karig, R. Weiss, Synthetic biology: new engineering rules for an emerging discipline, Mol Syst Biol, Vol. 2, msb4100073-E1msb4100073-E14 (2006).

[2] A. Becksei, L. Serrano, Engineering stability in gene networks by autoregulation, Nature, 405:590-593 (2000).

[3] C. Belta, L.C.G.J.M. Habets, Constructing decidable hybrid systems with velocity bounds, 43rd IEEE Conference on Decision and Control, pp. 467-472 (2004).

[4] C. Belta, L. Habets, V. Kumar, Control of multi-affine systems on rectangles with applications to hybrid biomolecular networks, 41st IEEE Conference on Decision and Control, pp. 534-539 (2002).

[5] G. Batt, D. Ropers, H. de Jong, J. Geiselmann, R. Mateescu, M. Page, D. Schneider, Validation of qualitative models of genetic regulatory networks by model checking: Analysis of the nutritional stress response in Escherichia coli, Bioinformatics, 21(Suppl 1):i19-i28, (2005). 
[6] G. Batt, D. Ropers, H. de Jong, J. Geiselmann, M. Page, D. Schneider, Qualitative analysis and verification of hybrid models of genetic regulatory networks : Nutritional stress response in Escherichia coli, HSCC 2005, LNCS 3414, pp. 134-150 (2005).

[7] J.L. Cherry, F.R. Adler, How to make a biological switch, J. Theor. Biol. 203:117-133 (2000).

[8] H. de Jong, J. Geiselmann, G. Batt, C. Hernandez, M. Page, Qualitative simulation of the initiation of sporulation in Bacillus subtilis, Bull. Math. Biol., 66(2):261-300 (2004).

[9] S. Drulhe, G. Ferrari-Trecate, H. de Jong, A. Viari, Reconstruction of switching thresholds in piecewise-affine models of genetic regulatory networks, HSCC 2006, LNCS 3927, pp. 184-199 (2006).

[10] R. Edwards, H.T. Siegelmann, K. Aziza, L. Glass, Symbolic dynamics and computation in model gene networks, Chaos, 11(1):160-169 (2001).

[11] T. Eißing, H. Conzelmann, E.D. Gilles, F. Allgöwer, E. Bullinger and P. Scheurich, Bistability analyses of a caspase activation model for receptor-induced apoptosis, J. Biol. Chem. 279(35):36892-36897 (2004).

[12] N. H. El-Farra, A. Gani, P. D. Christofides, Analysis of Mode Transitions in Biological Networks, AIChE J., 51, 2220-2234 (2005).

[13] M.B. Elowitz, S. Leibler, A synthetic oscillatory network of transcriptional regulators, Nature, 403:335-338 (2000).

[14] E. Farcot, Some geometric properties of piecewise affine biological network models, J. Math. Biol, 52(3):373-418 (2006).

[15] E. Farcot, J.-L. Gouzé, How to control a biological switch: a mathematical framework for the control of piecewise affine models of gene networks, Research Report RR-5979, INRIA Sophia-Antipolis, https://hal.inria.fr/ inria-00094853 (2006).

[16] T.S. Gardner, C.R. Cantor, J.J. Collins, Construction of a genetic toggle switch in Escherichia Coli, Nature, 403:339342 (2000)

[17] L. Glass, Combinatorial and topological methods in nonlinear chemical kinetics, J. Chem. Phys. 63:1325-1335 (1975).

[18] J.L. Gouzé, T. Sari, A class of piecewise linear differential equations arising in biological models, Dynamical systems, 17:299-316 (2003).

[19] L.C.G.J.M. Habets, J.H. van Schuppen, A control problem for affine dynamical systems on a full-dimensional polytope, Automatica 40, 21 - 35 (2004).

[20] J. Hasty, F. Isaacs, M. Dolnik, D. McMillen, J.J. Collins, Designer gene networks: towards fundamental cellular control, Chaos 11, 207-220 (2001).

[21] F.J. Isaacs, D.J. Dwyer, J.J. Collins, RNA synthetic biology, Nature Biotechnology 24, 545-554 (2006)

[22] F.J. Isaacs, D.J. Dwyer, C. Ding, D.D. Pervouchine, C.R. Cantor, J.J. Collins, Engineered riboregulators enable posttranscriptional control of gene expression, Nat Biotechnol 22: 841-847 (2004).

[23] M.K.J. Johansson, Piecewise Linear Control Systems, Springer, 2004.

[24] H. Kobayashi, M. Kaern, M. Araki, K. Chung, T.S. Gardner, C.R. Cantor, J.J. Collins, Programmable cells: interfacing natural and engineered gene networks, Proc. Natl. Acad. Sci. U.S.A., 101(22):8414-8419 (2004).

[25] T.J. Perkins, M. Hallett, L. Glass, Inferring models of gene expression dynamics, J. Theor. Biol. 230(3):289-299 (2004).
[26] D. Ropers, H. de Jong, M. Page, D. Schneider, J. Geiselmann, Qualitative simulation of the carbon starvation response in Escherichia coli, BioSystems, 84(2):124-152 (2006).

[27] Z. Sun, S.S. Ge, Analysis and synthesis of switched linear control systems, Automatica 41(2), pp. 181-195 (2005).

[28] R. Thomas, R. D'Ari, Biological Feedback, CRC-Press, Boca Raton, Florida (1990). 